\title{
Comparison of Single Photon Emission Computerised Tomography (SPECT) with Planar Wholebody Bone Scan (WBBS) in Diagnosis of Solitary Vertebral Lesion in Non-Skeletal Malignancies in Terms of Diagnostic Accuracy
}

\author{
Zaigham Salim Dar1, Umer-i-Farooq ${ }^{1}$, Fida Hussain², Mehdi Raza3 ${ }^{2}$ Ali Jamal2 and Khurram Hayat Mir² \\ ${ }^{1}$ Department of Nuclear Cardiology, Armed Forces Institute of Cardiology, Rawalpindi, Pakistan \\ ${ }^{2}$ Nuclear Medical Centre, Armed Forces Institute of Pathology, Rawalpindi, Pakistan \\ ${ }^{3}$ Department of Nuclear Cardiology, Army Cardiac Centre, Lahore, Pakistan
}

\begin{abstract}
Objective: To compare single photon emission computed tomography (SPECT) with planar whole body bone scan (WBBS) in diagnosis of solitary vertebral lesion in non-skeletal malignancies in terms of diagnostic accuracy.

Study Design: Cross-sectional study.

Place and Duration of Study: Nuclear Medical Centre, Armed Forces Institute of Pathology, Rawalpindi, July 2014 to June 2016.

Methodology: After fulfilling the inclusion and exclusion criteria and taking written informed consent, 74 patients of various extra-skeletal malignancies were enrolled in the study. Patients were injected with $740 \mathrm{MBq}$ of $99 \mathrm{mTc}$-methylene diphosphonate (MDP) intravenously. WBBS images were acquired in anterior and posterior projections 3 hours after injection. SPECT images of the desired vertebral region were acquired subsequently along with low dose CT. All the images were read by two experienced nuclear medicine physicians. Lesions were diagnosed as definitely benign, indeterminate/equivocal and definitely malignant separately on WBBS and SPECT. With SPECT/CT as gold standard, sensitivity, specificity, negative predictive value (NPV), positive predictive value (PPV) and accuracy were calculated.

Results: For planar WBBS, sensitivity was $91.43 \%$, NPV was $86.96 \%$, specificity was $51.28 \%$ and PPV remained $62.75 \%$. For SPECT, sensitivity and NPV were $100 \%$, specificity was $92.31 \%$ and PPV remained $92.11 \%$. Accuracy remained $70.27 \%$ and $95.95 \%$ for planar WBBS and SPECT, respectively.

Conclusion: SPECT imaging significantly increases the specificity $(p<0.05)$ of $99 \mathrm{mTC}-\mathrm{MDP}$ skeletal scintiscanning and accurately diagnoses the equivocal lesions as compared to planar images.
\end{abstract}

Key Words: Metastasis, WBBS, SPECT, SPECT/CT, 99mTc-MDP.

How to cite this article: Dar ZS, Farooq UI, Hussain F, Raza M, Jamal A, Mir KH. Comparison of single photon emission computerised tomography (SPECT) with planar wholebody bone scan (WBBS) in diagnosis of solitary vertebral lesion in non-skeletal malignancies in terms of diagnostic accuracy. J Coll Physicians Surg Pak 2019; 29(9):838-42.

\section{INTRODUCTION}

Diagnosis of distant metastasis is a mainstay in the staging of a malignancy as it determines the course of disease. ${ }^{1}$ Skeleton is one of the most common sites of distant metastasis. ${ }^{2}$ Most common site of skeletal metastasis is vertebral column (39\%), ${ }^{3}$ with highest predilection for lumbar spine $(52 \%)$, followed by dorsal $(36 \%)$ and the cervical spine (12\%). ${ }^{4}$ However, it is imperative to rule out coexisting benign etiologies like those in disc, facet joints and pars inter articularis and other sports injuries. ${ }^{5}$

Correspondence to: Dr. Zaigham Salim Dar, Department of

Nuclear Cardiology, Armed Forces Institute of Cardiology,

Rawalpindi, Pakistan

E-mail: xaighamaster@gmail.com

Received: December 13, 2017; Revised: May 12, 2019;

Accepted: June 14, 2019
Among conventional techniques, plain X-ray can only detect the lesion when there is loss of at least $50 \%$ bone substance with an overall sensitivity of around $50 \%{ }^{1}$ Presently, WBBS is the investigation of choice for detection of skeletal metastasis, followed by SPECT of indeterminate lesions. Further investigation of equivocal lesions is done with plain X-rays, CT and MRI. ${ }^{6}$ CT is highly sensitive for both osteolytic and osteoblastic lesions but not for marrow lesions. Magnetic resonance imaging (MRI) is a high contrast and high resolution technique for localisation of skeletal metastasis including that of bone marrow and their soft tissue extension. ${ }^{3}$

SPECT/CT is a well-established hybrid technique that can characterise skeletal lesions as definitely benign or definitely malignant instead of indeterminate or equivocal. 7 However, it puts increased radiation burden on patient in addition to extra cost and issue of nonavailability in our setup. $740 \mathrm{MBq}$ of $99 \mathrm{mTc}-\mathrm{MDP}$ used for 
WBBS or SPECT imaging exposes the patient to 4.2 to $5.2 \mathrm{mSv}$ with an additional dose of 3.8 to $4.2 \mathrm{mSv}$ for the CT component. 8

The rationale of this study was to ascertain superiority, or otherwise, of SPECT over WBBS imaging in localisation of vertebral metastasis with SPECT/CT as gold standard. Furthermore, objective of the study was to determine diagnostic accuracy of SPECT imaging in comparison with SPECT/CT, and subsequently employing the technique in metastatic workup of equivocal or indeterminate skeletal lesions, thus saving the cost and radiation burden to the patient along with overcoming the issue of non-availability of SPECT/CT.

\section{METHODOLOGY}

This cross-sectional study was carried out at Nuclear Medical Centre, AFIP, Rawalpindi, from July 2014 to June 2016 after approval from Hospital Ethical Committee. Through non-probability consecutive sampling, 74 patients, who fulfilled inclusion and exclusion criteria, were included after written informed consent. Patients of both genders with extra-skeletal malignancy reporting for metastatic workup and having a solitary vertebral lesion on planar WBBS were included in the study. Patients having characteristic multiple hot lesions on WBBS were excluded. Moreover, patients with history of previous chemotherapy or radiation therapy, neoadjuvant therapy, allergic to bispho-sphonates were also excluded from the study.

All patients were given $740 \mathrm{MBq}(20 \mathrm{mCi})$ 99mTc-MDP (PINSTECH) intravenously. Patients were advised plenty of liquids and frequent urination post injection. Images were acquired at 3 hours post-injection with

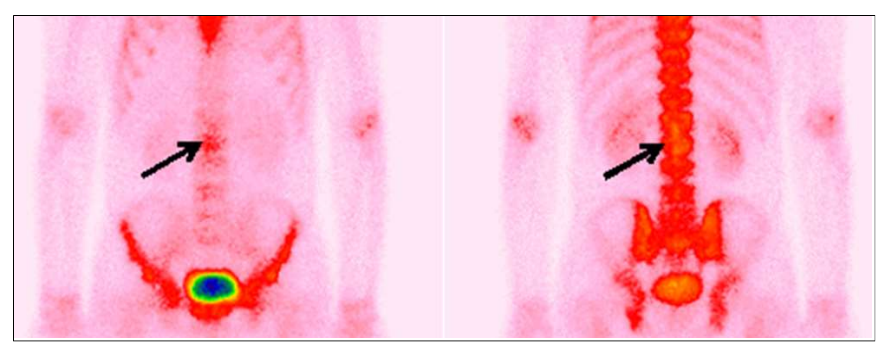

Figure 1: Anterior \& posterior planar images of lumbar spine showing solitary photon-rich focus (black arrowheads). hybrid SPECT/CT Siemens Symbia T6 camera. WBBS images were acquired with energy window of $20 \%$ around photopeak of $140 \mathrm{keV}$, using low energy high resolution (LEHR) collimator and $1024 \times 256$ matrix at a speed of $12 \mathrm{~cm}$ per minute. It was followed by SPECT with same energy window and collimator with matrix size of $256 \times 256.64$ projection images were acquired in circular orbit with 30 sec/image acquisition time. Low dose CT was acquired with $130 \mathrm{Kv}, 100 \mathrm{mAs}$, pitch of 1 , and a $512 \times 512$ matrix. Standard filters were applied and reconstruction was done with filtered back projection.

Images were read by two experienced nuclear medicine physicians (with at least 5 years experience). Lesions were classified as definitely benign, equivocal and definitely malignant on both planar WBBS and SPECT. Diagnosis was confirmed on SPECT/CT. The criteria used for diagnosis of benign/malignant lesion on SPECT/CT are summarised in Table I. For the purpose of calculating sensitivity, specificity, NPV, PPV and accuracy, equivocal lesions were considered as malignant.

The data was analysed using SPSS version 20 . Frequencies and percentages were calculated for qualitative variables like gender, type of tumour and location of metastasis. Mean + SD was calculated for quantitative data like age. In addition, frequencies with percentages were calculated for all categorical variables including sensitivity, specificity, negative predictive value, positive predictive value and accuracy with $2 \times 2$ contingency tables for WBBS and SPECT separately. Comparison between the two techniques was done using independent t-test, level of significance set at $p<0.05$.

\section{RESULTS}

A total of 74 patients were analysed with ages ranging from 31 years to 77 years $(58.66 \pm 10.11$ years) with $45.94 \%$ (34) females and $54.05 \%$ (40) males. Thirty-two $(43.2 \%)$ patients had carcinoma prostate followed by carcinoma breast in $37.8 \%(28)$, lung $(9.5 \%)$, renal cell $(5.4 \%)$, medullary thyroid $(2.7 \%)$ and colorectal $(1.4 \%)$ carcinomas. Fifty-one $(68.9 \%)$ patients had lesion identified in lumbar region, $25.7 \%$ (19) in thoracic spine, $4.1 \%(3)$ in sacrum, and $1.4 \%$ (1) had lesions in cervical

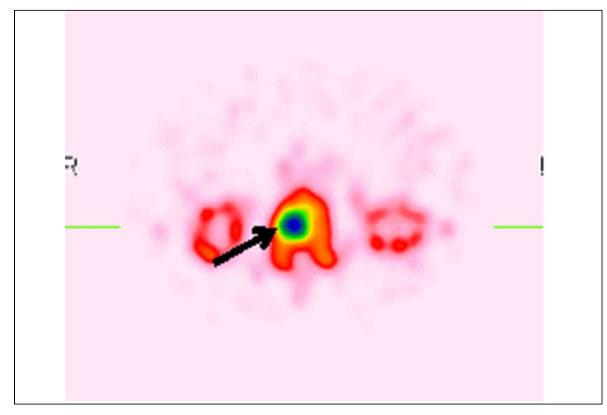

Figure 2: SPECT at LV2 showing photon-rich focus in vertebral body ( black arrowhead).

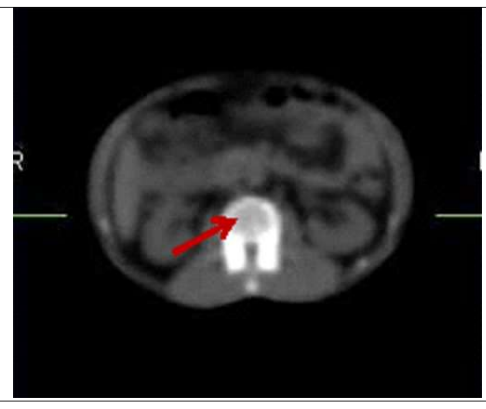

Figure 3: CT at LV2 showing a lytic lesion (red arrowhead).

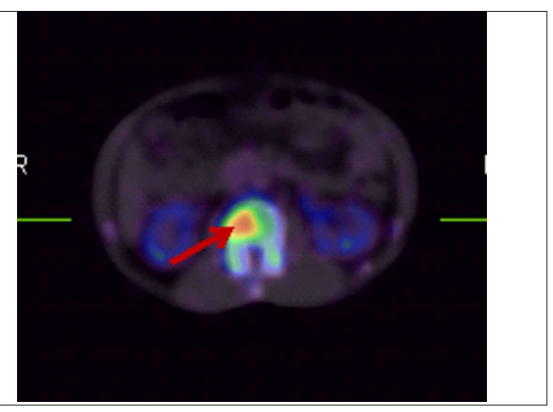

Figure 4: Fused SPECT/CT showing a metastatic lesion (red arrowhead). 
Table I: Diagnostic criteria on SPECT/CT.

\begin{tabular}{l|l}
\hline Benign & Malignant \\
\hline Facet joint & Body \\
Costovertebral joint & Pedicle \\
Transverse & Cold lesion \\
Spinous process & Lytic lesion \\
Adjacent vertebra disc & \\
\hline
\end{tabular}

Table II: Comparison of planar WBBS vs. SPECT/CT.

\begin{tabular}{l|c|c|c}
\hline \multirow{2}{*}{ WBBS } & \multicolumn{2}{|c|}{ SPECT/CT } & \multirow{2}{*}{} \\
\cline { 2 - 3 } Malignant & Malignant & Benign & \\
& 32 & 19 & PPV \\
& & & $62.75 \%$ \\
\hline Benign & 3 & 20 & NPV \\
& & & $86.96 \%$ \\
\hline & SEN & SPC & ACC \\
& $91.43 \%$ & $51.28 \%$ & $70.27 \%$ \\
\hline
\end{tabular}

WBBS: Whole-body bone scan; SPECT: Single photon emission computerised tomography; CT: Computerised tomography; MRI: Magnetic resonance imaging; PET: Photon emission tomography; MDP: Methylene diphosphonate; ${ }^{99 m} T c$ : Technetium - 99m.

Table III: Comparison of SPECT vs. SPECT/CT.

\begin{tabular}{l|c|c|c}
\hline \multirow{2}{*}{ SPECT } & \multicolumn{2}{|c|}{ SPECT/CT } & \\
\cline { 2 - 3 } Malignant & Malignant & Benign & \\
\hline Benign & 35 & 3 & PPV \\
& 0 & 36 & NPV \\
& & & $100.00 \%$ \\
\hline & SEN & SPC & ACC \\
& $100.00 \%$ & $92.31 \%$ & $95.95 \%$ \\
\hline
\end{tabular}

WBBS: Whole-body bone scan; SPECT: Single photon emission computerised tomography; CT: Computerised tomography; MRI: Magnetic resonance imaging; PET: Photon emission tomography; MDP: Methylene diphosphonate; ${ }^{99 m} T c:$ Technetium - 99m.

spine. On SPECT/CT $66.2 \%$ (49) had lesion in vertebral body; out of which, 5 lesions were lytic and 44 were sclerotic. This was followed by facet joint which had lesion in $16.2 \%$ (12) patients. Rest of the patients had lesions identified in pedicle $(9.5 \%)$, disc $(5.4 \%)$ and transverse and spinous processes $(2.8 \%)$.

Planar WBBS images showed that lesions were benign in 23 patients, indeterminate in 36 , and malignant in 15 patients. SPECT images demonstrated that lesions in 36 patients were benign, 8 were indeterminate, and 30 were malignant. Standard evaluation of SPECT/CT images revealed benign lesions in 39 patients and malignant lesions in 35 patients. The results are summarised in Table II.

Out of 36 indeterminate lesions identified on planar WBBS, $41.66 \%$ (15) were diagnosed as benign on SPECT/CT while rest of the $58.33 \%$ (21) were malignant. Similarly, out of 8 indeterminate lesions identified on SPECT, $25 \%$ (2) were diagnosed as benign on SPECT/CT and $75 \%$ (6) as malignant, as shown in Table III. Moreover, 20\% (3/15) patients diagnosed as malignant on WBBS were found benign on SPECT/CT while only $3.3 \%(1 / 30)$ evaluated as malignant on SPECT were diagnosed as benign on SPECT/CT. In addition, $8.7 \%(2 / 23)$ lesions declared benign on WBBS were diagnosed malignant on SPECT/CT while no such case was noted in case of SPECT.

Based on these results, for planar WBBS, sensitivity was 91.43\% NPV $86.96 \%$, specificity $51.28 \%$ and PPV was $62.75 \%$. For SPECT, sensitivity and NPV were $100 \%$, specificity was $92.31 \%$ and PPV remained $92.11 \%$. Accuracy remained $70.27 \%$ and $95.95 \%$ for planar WBBS and SPECT, respectively $(p=0.04)$.

\section{DISCUSSION}

Skeletal scintigraphy with $99 \mathrm{mTc}$ labelled bisphosphonates is a highly sensitive technique for detection of skeletal metastasis that can scan complete skeleton at reasonable cost. ${ }^{9}$ However, due to its low specificity, a solitary focal lesion in spine seen on WBBS has $60-70 \%$ probability of being metastatic. 10 SPECT adds to the utility of scintigraphy as it improves target to background ratio and the image contrast which improves diagnostic specificity. ${ }^{11}$ Incorporation of anatomic imaging like CT further adds to the diagnostic accuracy of SPECT. ${ }^{12}$

Addition of SPECT/CT to WBBS significantly enhances sensitivity and specificity while lowering the proportion of indeterminate diagnosis. However, the most important outcome is the modification in patient management plan by up or down staging of disease by more precisely identifying a metastatic lesion. ${ }^{13}$ In addition to characterising indeterminate skeletal lesions by accurately demarcating anatomy of pedicles, vertebral bodies, facet joint and intervertebral discs, SPECT/CT can identify lytic lesions, thus differentiating benign from malignant. ${ }^{7}$ Studies have shown that a lesion in pedicle has highest ( $87.7 \%$ to $100 \%$ ) probability to be malignant followed by vertebral body (36.8 - $57.3 \%$ ), spinous process (18.7 $81.3 \%)$ and facet joint $(0.8-21.4 \%) .{ }^{14}$

Although, PET and MRI have the ability to detect earliest metastatic infiltrations with quantification, yet their utility is limited, due to non-availability and high cost. ${ }^{15}$ FDG $\mathrm{PET} / \mathrm{CT}$ is more sensitive, specific and accurate technique for detection of skeletal metastases than conventional bone scans, especially in vertebral and pelvic lesions. ${ }^{16}$ However, PET/MRI supersedes $\mathrm{PET} / \mathrm{CT}$ with improved conspicuity in bone metastases detection, though overall detection rate remains same for the two. ${ }^{17}$

This study shows the advantage of SPECT over planar WBBS. Sensitivity and NPV remained $100 \%$ for SPECT and $91.43 \%$ and $86.96 \%$, respectively for WBBS proving both of them to be excellent screening tools. However, SPECT has shown much higher specificity and PPV, $92.31 \%$ and $92.11 \%$, respectively, as compared to $51.28 \%$ and $62.75 \%$, respectively for WBBS $(p=0.01)$. Diagnostic accuracy for WBBS and SPECT was $70.27 \%$ and $95.95 \%$, respectively $(p=0.04)$. In this study, for the purpose of calculation, we considered all equivocal cases as positive for malignancy. This enabled a 
sensitivity close to $100 \%$ but reduced the specificity significantly. SPECT considerably enhances the confidence with which a lesion is reported as definitely benign or malignant instead of indeterminate or equivocal. WBBS diagnosed nearly half (36/74) of the lesions as indeterminate which were reduced to $10.8 \%$ (8/74) with SPECT, thus reducing the cost of further investigation.

Comparing WBBS with SPECT/CT, 35.29\% (18/51) lesions considered as malignant (indeterminate cases included) on WBBS were evaluated as benign on SPECT/CT. In this regard, $41.66 \%$ (15/36) indeterminate lesions and $20.0 \%(3 / 15)$ definitely malignant lesions were diagnosed as benign, thus reducing the specificity of the test. Reasons behind these false diagnoses were intervertebral disc infections spreading to adjacent vertebral bodies, compression fractures, and facet joint pathologies. In addition $8.7 \%(2 / 23)$ patients that were found definitely benign on WBBS came out to be malignant on SPECT/CT due to presence of lytic lesion.

Comparing SPECT with SPECT/CT, only $7.9 \%(3 / 38)$ lesions considered as malignant (indeterminate lesions included) on SPECT were evaluated as benign on SPECT/CT. In this regard, $20.0 \%(2 / 8)$ indeterminate lesions and $3.3 \%(1 / 30)$ definitely malignant lesions were diagnosed as benign. Reasons behind these false diagnoses were intervertebral disc infections. Calculating the impact on management, treatment of more than $27.0 \%(20 / 74)$ patients undergoing only WBBS and less than $5 \%(3 / 74)$ of patients undergoing SPECT was altered by SPECT/CT.

Several studies have compared WBBS, SPECT, and SPECT/CT in equivocal lesions and have shown a substantial improvement in specificity with SPECT/CT. However, no significant local data has been published in this regard. Sedonja et al. compared 37 patients without known malignancy with 38 patients of malignancy. SPECT was able to detect 58/64 (90.63\%) lesions correctly while planar scintigraphy could only evaluate $42 /(65.63 \%)$ lesions $(p=0.01) .{ }^{18}$ Another study was done at University Hospital Zurich, where out of 37 patients, planar scintigraphy could only identify $64 \%$ lesions correctly while SPECT diagnosed $86 \%$ with $100 \%$ result on SPECT/CT.19 Compared to these results, in this study, $52 / 74(70.27 \%)$ were diagnosed correctly by WBBS and $71 / 74(95.95 \%)$ patients were correctly diagnosed on SPECT/CT.

Talbot et al. showed sensitivity and specificity of $78 \%$ and $48 \%$, respectively for WBBS; and $87 \%$ and $91 \%$, respectively for SPECT.20 Compared to this, sensitivity and specificity of WBBS were $91 \%$ and $51 \%$ while those of SPECT were $100 \%$ and $92 \%$ in this study.

Review of recent meta-analysis done on comparison of various other diagnostic modalities for skeletal metastasis have revealed spectrum of results with varying relations between sensitivities, specificities and accuracies of different modalities. Liu et al. showed that on per-patient basis, sensitivity of SPECT was comparable with $\mathrm{PET}$; and MRI and was significantly higher than that of WBBS and CT. However, it showed specificity of WBBS that was not significantly different from MRI and CT, but higher than SPECT and even PET. Diagnostic odd ratios (DORs) were better for SPECT than WBBS. However, MRI was the best and CT the lowest. ${ }^{21}$

In another recent meta-analysis, Yang et al. found that, on per-patient basis, the pooled sensitivity estimates for scintigraphy $(86.0 \%)$ was significantly better than CT $(72.9 \%)$. However, both were significantly lower than PET (89.7\%) and MRI (90.6\%). On the contrary, the pooled specificity estimates for scintigraphy $(81.4 \%)$ was significantly lower than CT (94.8\%), MRI (95.4\%) and PET (96.8\%). However, on per-lesion basis, the pooled sensitivity estimates for scintigraphy $(75.1 \%)$ were not significantly different from CT $(77.1 \%)$ but both were significantly lower than MRI $(90.4 \%)$ and PET (86.9\%). Similarly, the pooled specificity estimates for scintigraphy $(93.6 \%)$ was higher than CT $(83.2 \%)$, but lower than MRI (96.0\%) and PET (97.0\%). ${ }^{22}$

There were a few imitations in this study. Sample size was considerably small with very few patients having lytic lesions. Since lytic lesions were major cause of false negatives on WBBS, more patients with tumors that produce lytic pathologies could have affected the results. Therefore, it is recommended that patients with such tumors should undergo SPECT or preferably SPECT/CT during their metastatic workup. However, those tumors that produce osteoblastic metastasis can be assessed by WBBS aided with SPECT.

\section{CONCLUSION}

WBBS and SPECT are highly sensitive techniques for detection of skeletal metastasis. However, WBBS with its low specificity becomes unable to differentiate between metastatic and concurrently occurring benign lesion in vertebral column. SPECT, on the other hand, has reasonably high specificity and can be employed as diagnostic tool for vertebral metastasis instead of SPECT/CT, which remains the gold standard.

\section{ETHICAL APPROVAL:}

This cross-sectional study was carried out at Nuclear Medical Centre, AFIP after approval from Hospital Ethical Committee.

\section{CONFLICT OF INTEREST:}

No conflict of interest, financial or otherwise, has been involved in carrying out this research.

\section{AUTHORS' CONTRIBUTION:}

ZSD: Conception and design of study, acquisition of data, 
statistical analysis of data, drafting of manuscript, final approval.

UIF: Concept and design of study, statistical analysis of data, drafting and revising the manuscript, final approval. FH, MR: Data interpretation (interpretation of scans), revision of manuscript, final approval.

AJ, KHM: Acquisition of data, statistical analysis of data, drafting of manuscript (literature review), final approval.

\section{REFERENCES}

1. Walter W, Gübitz R, Vieth V, Weckesser M, Schober $O$, Schäfers M. The diagnostic imaging of bone metastases. Dtsch Arztebl Int 2014; 111:741-7.

2. Disibio G, French SW. Metastatic patterns of cancer: Results from a large autopsy study. Arch Pathol Lab Med 2008; 132: 931-9.

3. Taoka T, Mayr NA, Lee HJ, Yuh WT, Simonson TM, Rezai K, et al. Factors influencing visualization of vertebral metastases on MR imaging versus bone scintigraphy. AJR Am J Roentgenol 2001; 176:1525-30.

4. O'Sullivan GJ, Carty FL, Carmel G, Cronin CG. Imaging of bone metastasis: An update. World J Radiol 2015; 7:202-11.

5. Sudhakar P, Sharma AR, Bhushan SM, Ranadhir G, Narsimuhulu G, Rao VVSP. Efficacy of SPECT over planar bone scans in the diagnosis of solitary vertebral lesions in patients with low back pain. Indian J Nucl Med 2010; 25:44-8.

6. Saha S, Burke C, Desai A, Vijayanathan S, Gnanasegaran G. SPECT-CT: Applications in musculoskeletal radiology. $\mathrm{Br} J$ Radiol 2013; 86:20120519.

7. Ghosh P. The role of SPECT/CT in skeletal malignancies. Semin Musculoskelet Radiol 2014; 18:175-93.

8. Ferrari M, De Marco P, Origgi D. Pedroli G. SPECT/CT radiation dosimetry. Clin Transl Imaging 2014; 2:557-69.

9. Sharma P, Dhull VS, Reddy RM, Bal C, Thulkar S, Malhotra A, et al. Hybrid SPECT-CT for characterizing isolated vertebral lesions observed by bone scintigraphy: Comparison with planar scintigraphy, SPECT, and CT. Diagn Interv Radiol 2013; 19:33-40.

10. Zeissman HA, O'Malley JP, Thrall JH. Nuclear medicine: The requisites. 4th ed. Philadelphia Elsevier Saunders; 2014.

11. Iqbal B, Currie GM, Wheat JM, Raza H, Kiat H. The incremental value of SPECT/CT in characterizing solitary spine lesions. J Nucl Med Technol 2011; 39:201-7.
12. Yiqiu Z, Hongcheng S, Dengfeng C, Lei J, Yan X, Beilei L, et al. Added value of SPECT/spiral CT versus SPECT in diagnosing solitary spinal lesions in patients with extra-skeletal malignancies. Nucl Med Commun 2013; 34:451-8.

13. Mahaletchumy $T, A b A z i z A$. Incremental value of single-photon emission computed tomography - computed tomography for characterization of skeletal lesions in breast cancer patients. World J Nucl Med 2017; 16:303-10.

14. Reinartz P, Schaffeldt J, Sabri O, Zimny M, Nowak B, Ostwald E, et al. Benign versus malignant osseous lesions in the lumbar vertebrae: Differentiation by means of bone SPET. Eur J Nucl Med 2000; 27:721-6.

15. Lecouvet FE, Talbot JN, Messiou C, Bourguet P, Liu Y, de Souza NM, EORTC Imaging Group. Monitoring the response of bone metastases to treatment with Magnetic Resonance Imaging and nuclear medicine techniques: A review and position statement by the European Organisation for Research and Treatment of Cancer imaging group. Eur $J$ Cancer 2014; 50:2519-31.

16. Chang CY, Gill CM, Simeone FJ, Taneja AK, Huang AJ, Torriani M, et al. Comparison of the diagnostic accuracy of $99 \mathrm{~m}$-Tc-MDP bone scintigraphy and 18 F-FDG PET/CT for the detection of skeletal metastases. Acta Radiol 2016; 57:58-65.

17. Samarin A, Hüllner M, Queiroz MA, Stolzmann $P$, Burger IA, von Schulthess G, et al. 18F-FDG-PET/MR increases diagnostic confidence in detection of bone metastases compared with 18F-FDG-PET/CT. Nucl Med Commun 2015; 36:1165-73.

18. Sedonja I. The benefit of SPECT when added to planar scintigraphy in patients with bone metastases in the spine. Clin Nucl Med 1999; 24:407-13.

19. Strobel K, Burger C, Seifert B, Husarik DB, Soyka JD, Hany TF. Characterization of focal bone lesions in the axial skeleton: performance of planar bone scintigraphy compared with SPECT and SPECT fused with CT. AJR Am J Roentgenol 2007; 188:W467-74

20. Talbot JN, Paycha F, Balogova S. Diagnosis of bone metastasis: Recent comparative studies of imaging modalities. $Q J$ Nucl Med Mol Imaging 2011; 55:374-410.

21. Liu T, Wang S, Liu H, Meng B, Zhou F, He F, et al. Detection of vertebral metastases: A meta-analysis comparing MRI, CT, PET, BS and BS with SPECT. J Cancer Res Clin Oncol 2017; 143:457-65

22. Yang HL, Liu T, Wang XM, Xu Y, Deng SM. Diagnosis of bone metastases: A meta-analysis comparing ${ }^{18} \mathrm{FDG}$ PET, CT, MRI and bone scintigraphy. Eur Radiol 2011; 21:2604-17. 